\title{
Mécanismes de l'effet diurétique de la caféine
}

$>$ La caféine est un psychotrope naturel abondamment consommé. Outre ses propriétés psychostimulantes, elle exerce un effet diurétique dont les mécanismes ne sont pas clairement établis. Une revue de la littérature indique que l'effet diurétique de la caféine implique l'antagonisme des récepteurs $\mathrm{Al}$ de l'adénosine à différents niveaux : (1) la caféine augmente le taux de filtration glomérulaire par inhibition de la vasoconstriction de l'artériole afférente lors du réflexe tubulo-glomérulaire; (2) la caféine inhibe la réabsorption sodée par le tube contourné proximal, ce qui augmente la diurèse ; et (3) la caféine inhibe le réflexe hépatorénal via les terminaisons nerveuses des espaces de Mall. <

La caféine est un alcaloïde (1,3,7-triméthylxanthine) de la famille des méthylxanthines que l'on retrouve dans les graines, feuilles et fruits de diverses plantes où elle agit comme insecticide naturel. La caféine, présente dans le café, est obtenue par infusion à partir de graines de caféier, mais on en trouve également dans les fèves de chocolat, les feuilles de thé, les graines de guarana et la noix de cola. Les boissons caféinées les plus consommées sont le café $(85 \mathrm{mg}$ par tasse de $150 \mathrm{ml}$ à partir de grains de café torréfié moulu, ou $60 \mathrm{mg} / 150 \mathrm{ml}$ pour un café instantané), le thé (30 mg par feuille/sachet ou $20 \mathrm{mg}$ par tasse s'il s'agit de thé instantané) et, dans une moindre mesure, le cacao. La caféine est également un ingrédient commun à diverses boissons énergisantes, comme le Coca-Cola ${ }^{\circledR}$ $(\sim 10 \mathrm{mg} / 100 \mathrm{ml})$ et le Red Bull ${ }^{\oplus}(\sim 24 \mathrm{mg} / 100 \mathrm{ml})$. On la trouve aussi dans certains médicaments, principalement en association avec le paracétamol ou l'acide acétylsalicylique dans des antalgiques/antipyrétiques (Antigrippine ${ }^{\oplus}$, Perdolan Compositum ${ }^{\oplus}$ ), mais également en association avec l'ergotamine (Cafergot ${ }^{\oplus}$, un anti-migraineux). En Europe, les adultes consomment en moyenne $200 \mathrm{mg}$ de caféine par jour, essentiellement

Vignette (๔) Image adaptée par François Jouret au départ de http://campus. cerimes.fr).

\section{Barbara Marx ${ }^{1}$, Éléonore Scuvée ${ }^{1}$, Jacqueline Scuvée-Moreau ${ }^{2}$, Vincent Seutin ${ }^{2}$, François Jouret ${ }^{3,4}$}

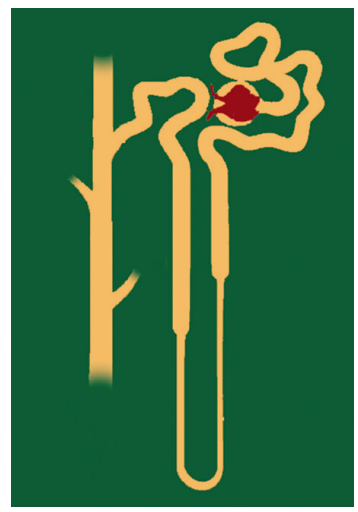

${ }^{1}$ Faculté de médecine, université de Liège, 4000 Liège, Belgique; ${ }^{2}$ Service de pharmacologie et laboratoire de neurophysiologie, GIGA neurosciences, université de Liège, 4000 Liège, Belgique ; ${ }^{3}$ GIGA cardiovasculaires, université de Liège, Avenue Hippocrate, 13, 4000 Liège, Belgique; ${ }^{4}$ Service de néphrologie, CHU de Liège, 4000 Liège Belgique. francois.jouret@chu.ulg.ac.be

sous forme de café, de thé et de boissons énergisantes. Les Pays-Bas et les pays nordiques tels que le Danemark, la Finlande, la Norvège et la Suède, sont les plus friands de caféine, avec une consommation quotidienne moyenne atteignant $400 \mathrm{mg}$ [1].

Du point de vue pharmacologique, la caféine exerce ses principaux effets par l'intermédiaire d'un antagonisme compétitif des récepteurs de l'adénosine, récepteurs couplés aux protéines $G$ dont il existe plusieurs types : Al (couplés aux protéines Gi/o : la stimulation de ce récepteur entraîne une inhibition de l'adénylate cyclase $[A C]$, une ouverture de canaux potassium $\left[\mathrm{K}^{+}\right]$et une fermeture de canaux calcium $\left[\mathrm{Ca}^{2+}\right]$ ); A2a et A2b (couplés aux protéines Gs : la stimulation de ces récepteurs entraîne une activation de l'AC) ; $A 3$ (couplés aux protéines $\mathrm{Gi} / 0$ ). Les récepteurs $\mathrm{Al}$ et $\mathrm{A} 2$ sont largement distribués dans l'organisme, notamment au niveau du système nerveux central (SNC), des bronches, des vaisseaux, du cœur et des reins. Ils participent à de multiples fonctions physiologiques et pathologiques [2]. L'effet antagoniste de la caféine au niveau du récepteur $\mathrm{Al}$ conduit à une stimulation de l'AC, tandis que l'antagonisme, au niveau du récepteur A2, l'inhibe. Ces effets entraînent des variations qui correspondent à des modifications des taux intracellulaires d'adénosine mono-phosphate cyclique (AMPc). La caféine est également un inhibiteur compétitif mais non spécifique des phosphodiestérases (qui dégradent l'AMPc). Cet effet semble cependant négligeable aux concentrations plasmatiques habituelles de caféine. Enfin, d'autres effets cellulaires de la caféine ont été décrits, comme la libération $\mathrm{du}^{\mathrm{Ca}^{2+}}$ à partir des réservoirs intracellulaires via une interaction avec les canaux calciques sensibles à la ryanodine ${ }^{1}$, et l'augmentation de la libération de

\footnotetext{
${ }^{1}$ Les récepteurs de la ryanodine, RyR, sont sensibles également à la caféine.
} 


\begin{tabular}{|c|c|c|c|c|}
\hline Récepteur & Protéine G & Organes & Effets de l'adénosine & Antagonisme par la caféine \\
\hline \multirow{8}{*}{ Al } & $\mathrm{Gi} / 0$ & SNC & y Libération de divers neu- & Psychostimulant \\
\hline & $-\boldsymbol{y}[A M P c]$ & & rotransmetteurs & Y Somnolence \\
\hline & $-(+)$ Canaux $\mathrm{K}^{+}$ & & Sédation & $\boldsymbol{\lambda}$ Attention \\
\hline & - (-) Canaux $\mathrm{Ca}^{2+}$ & Cœur & Chronotrope (-) & Inotrope (+) \\
\hline & & & Inotrope (-) & Chronotrope (+) \\
\hline & & Vaisseaux & Vasoconstriction & Vasodilatation \\
\hline & & a. afférente rénale & & \\
\hline & & Système respiratoire & $\begin{array}{l}\text { Bronchoconstriction } \\
\text { chez asthmatiques }\end{array}$ & Bronchodilatation \\
\hline \multirow{11}{*}{ A2 } & Gs & SNC & $\boldsymbol{\lambda}$ Libération de divers neu- & (+) Centre bulbaire de la respiration \\
\hline & $-\boldsymbol{\lambda}[\mathrm{AMPc}]$ & & rotransmetteurs & (+) Transmission D2 \\
\hline & & & & (+) Dépendance \\
\hline & & Système vasculaire & Vasodilatation & Vasoconstriction \\
\hline & & a. coronaire & & Effet anti-migraineux \\
\hline & & a. cérébrale & & \\
\hline & & a. efférente rénale & & \\
\hline & & vaisseaux & & \\
\hline & & squelettiques & & \\
\hline & & Système digestif & (-) Sécrétions & (+) Sécrétions \\
\hline & & & & Relaxation du SOI \\
\hline
\end{tabular}

Tableau I. Principaux effets liés à la stimulation des récepteurs A1 et A2 à l'adénosine et effets de la caféine. SNC : système nerveux central ; SOI : sphincter œsophagien inférieur ; (+) : stimulation ; (-) : inhibition ; a. : artère ; D2 : récepteur dopaminergique de type D2.

catécholamines par les glandes surrénales. Dans ces cas, également, il s'agit probablement d'effets «supra-pharmacologiques », les effets de libération $\mathrm{du} \mathrm{Ca}^{2+}$ ne se produisant qu'à partir de concentrations millimolaires.

La caféine peut influencer le fonctionnement de divers organes selon les récepteurs qu'ils expriment et du degré d'activation de ceux-ci en conditions physiologiques ou pathologiques (Tableau I).

Au niveau du SNC, la caféine exerce un effet psychostimulant via l'antagonisme du récepteur Al. Cette action diminue la somnolence et augmente l'attention mais aussi, parfois, l'anxiété. Les récepteurs Al sont situés sur des terminaisons aminergiques (liées à la libération de noradrénaline, de dopamine et de sérotonine) où ils inhibent toniquement la libération de ces neuromodulateurs. La caféine augmente donc, par exemple, la libération de noradrénaline dans le cortex cérébral, avec pour conséquence son activation. À doses faibles ou modérées (c'est-à-dire moins de 4 tasses de café par jour), la caféine a des propriétés antimigraineuses, probablement en antagonisant les effets de l'adénosine sur les récepteurs A2 vasodilatateurs qui sont présents au niveau des muscles lisses vasculaires. De plus, la caféine potentialise l'effet de certains analgésiques communément utilisés, comme le paracétamol et l'ibuprofène. Ces propriétés pro-analgésiques s'expliquent par une action à différents niveaux des voies de transmission de la douleur [3]. Des interactions complexes existent entre les récepteurs de l'adénosine $\mathrm{Al}$ et $\mathrm{A} 2$ et la transmission dopaminergique. Ainsi, l'antagonisme du récepteur A2a par la caféine favorise la transmission dopaminergique $D 2^{2}$, ce qui expliquerait ses possibles effets bénéfiques dans la maladie de Parkinson [4]. Ces récepteurs A2a semblent également jouer un rôle dans les effets de renforcement positif de la caféine et la dépendance modérée qui lui est associée [5].

Au niveau cardiovasculaire, la caféine exerce un effet chronotrope positif par antagonisme du récepteur $\mathrm{Al}$, étant ainsi responsable d'une tachycardie symptomatique lors d'une ingestion de doses supérieures à $500 \mathrm{mg}$. Cet effet inotrope positif ${ }^{3} \mathrm{~s}^{\prime}$ explique par l'augmentation de l'entrée intracellulaire de $\mathrm{Ca}^{2+}$ durant le potentiel d'action [6]. Au niveau vasculaire, en fonction de la prédominance tissulaire des récepteurs $\mathrm{Al}$ ou des récepteurs $A 2$, la caféine peut provoquer soit une vasoconstriction, soit une vasodilatation [7].

\footnotetext{
${ }^{2}$ Les récepteurs dopaminergiques sont classés en deux familles D1 et D2 selon la nature de la protéine G à laquelle ils sont couplés (Gs pour D1, Gi pour D2).

${ }^{3}$ Une substance est inotrope positive si elle augmente l'amplitude des battements cardiaques.
} 


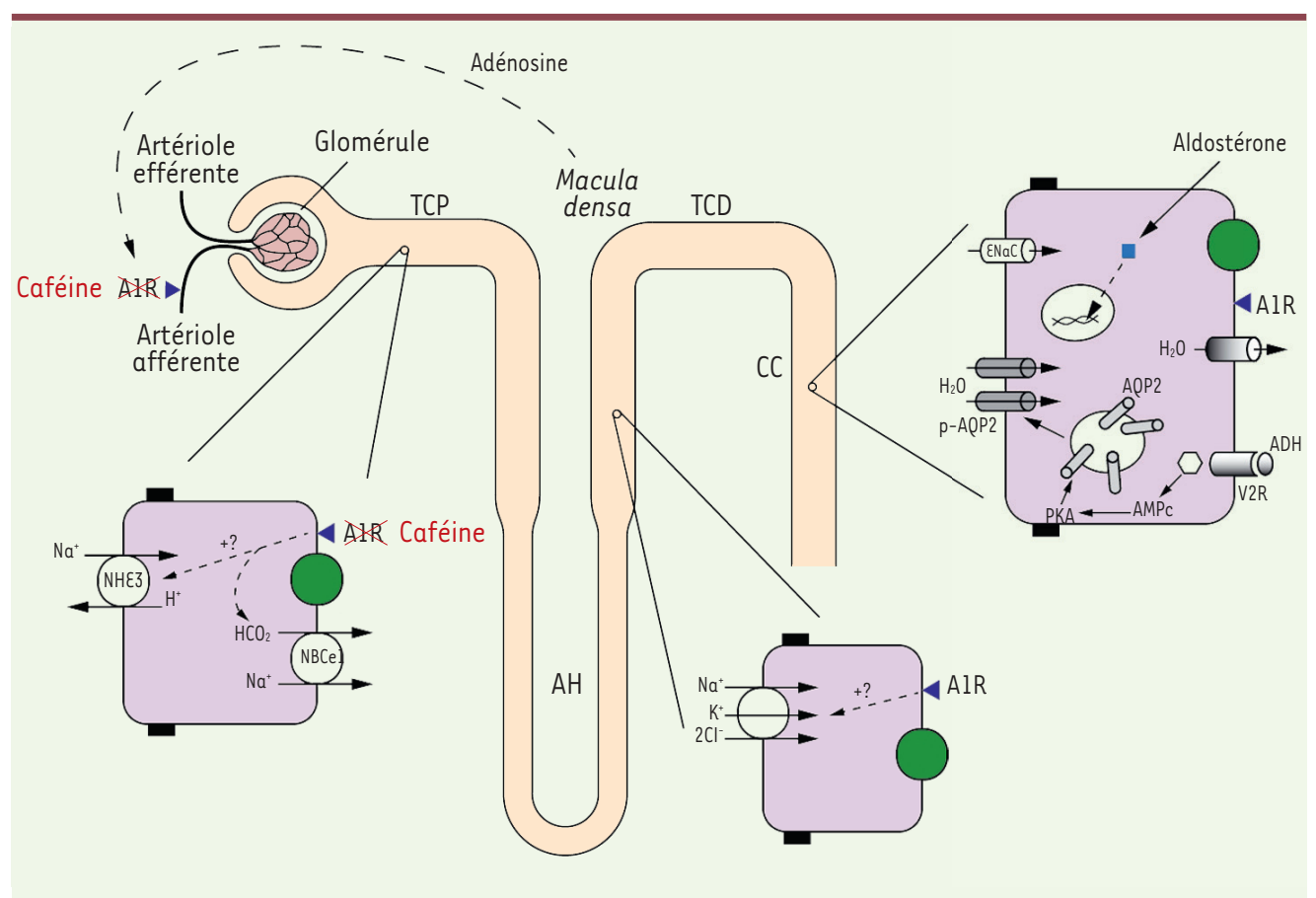

Figure 1. Représentation schématique des segments du néphron impliqués dans l'homéostasie hydro-sodée avec focus sur le contrôle exercé par la caféine. Au niveau du tube contourné proximal (TCP), la réabsorption de $\mathrm{Na}^{+}$ s'effectue par co-transport d'autres solutés ou par antiport d'ions $\mathrm{H}^{+}$via l'échangeur $\mathrm{Na}^{+} / \mathrm{H}^{+}$(NHع3), avec un rôle moteur de la pompe $\mathrm{Na}^{+} / \mathrm{K}^{+}-$ ATPase (rond vert). La réabsorption d'eau se fait par voie para- (jonctions serrées, rectangles noirs) et transcellulaire (via les canaux à eau, aquaporines $(A Q P)$ de type 1). La branche ascendante large de l'anse de Henlé $(\mathrm{AH})$, imperméable à l'eau, assure une résorption active de $\mathrm{Na}^{+}$via le cotransporteur $\mathrm{Na}^{+} / \mathrm{K}^{+} / 2 \mathrm{Cl}^{-}$( $\mathrm{NKCC} 2$ ). Au niveau des cellules principales du canal collecteur $(C C)$, la vasopressine $(A D H)$ augmente la perméabilité à $\mathrm{H}_{2} \mathrm{O}$ en régulant l'insertion des AQP2 (phosphorylée, $\mathrm{p}$-APP2) dans la membrane apicale, via le récepteur V2 (V2R). La résorption de $\mathrm{Na}^{+}$y est contrôlée par l'aldostérone (récepteur cytosolique, carré bleu). L'adénosine module la physiologie rénale via les récepteurs $\mathrm{Al}$. Elle stimule le transport du $\mathrm{Na}^{+}$au niveau du TCP, et intervient dans le rétrocontrôle tubulo-glomérulaire en entraînant une vasoconstriction de l'artériole afférente en réponse à une augmentation de la concentration en $\mathrm{Na}^{+}$au niveau de la macula densa du tube contourné distal (TCD). L'effet natriurétique et diurétique de la caféine s'explique par son action antagoniste sur les récepteurs Al. AlR : récepteur de l'adénosine Al ; ENaC: epithelial sodium channel; NBCel : electrogenic sodium bicarbonate cotransporter 1 ; PKA : protéine kinase A.

Au niveau du poumon, la caféine antagonise, chez les asthmatiques, l'effet bronchoconstricteur de l'adénosine, probablement par l'intermédiaire du récepteur $\mathrm{Al}$ [8]. Elle stimule également le centre bulbaire de la respiration justifiant son utilisation, de même que celle de la théophylline, pour la prévention de l'apnée du nouveau né prématuré. Dans le tractus gastro-intestinal, la caféine stimule les sécrétions digestives. Elle induit le relâchement des muscles lisses intestinaux et déprime le sphincter inférieur de l'œsophage. Ces effets pourraient expliquer la symptomatologie de reflux gastro-œsophagien décrite par certains consommateurs de café [9].

Enfin, la caféine est dotée d'effets métaboliques sur la glycogénolyse et la lipolyse au niveau des muscles squelettiques, des reins et du foie, avec pour résultat global, une amélioration des performances physiques [10]. Outre les effets systémiques de la caféine que nous avons brièvement mentionnés, la caféine exerce un effet diurétique significatif lors d'une ingestion de $300 \mathrm{mg}$, soit 4 à 5 tasses de café $[11,12]$. À partir de $650 \mathrm{mg}$ en prise aiguë, une perte hydrique totale de 2,7\% peut être observée chez l'adulte si ses apports en eau restent stables [13]. Le pouvoir diurétique de la caféine est d'autant plus grand que la balance hydrique de l'individu est positive et que le sujet est jeune et n'est pas habitué à la prise de café [14].

Après un bref rappel de l'homéostasie hydrique et une description du rôle complexe de l'adénosine à ce niveau, nous allons détailler les mécanismes physiologiques de cet effet diurétique induit par la caféine.

\section{Physiologie de l'homéostasie hydro-sodée et modulation par l'adénosine}

L'homéostasie de l'eau et du sel $(\mathrm{NaCl})$ implique tous les segments du néphron (Figure 1) [15]. Au niveau du tube contourné proximal (TCP), la réabsorption de l'eau se fait selon le gradient de $\mathrm{Na}^{+}$par voie paracellulaire et transcellulaire (via les canaux perméables à l'eau appelés « aquaporines » de type 1 [APPl]). L'absence d'expression d'AQPl chez l'homme ${ }^{4}$ n'a en effet de conséquence que dans des conditions extrêmes de restriction hydrique. Le passage $\mathrm{du} \mathrm{Na}^{+}$à travers la membrane apicale se fait grâce à un transport actif secondaire, par cotransport d'autres solutés, tels que les acides aminés, le phosphate, le glucose ou par antiport d'ions $\mathrm{H}^{+}$via l'échangeur $\mathrm{Na}^{+} / \mathrm{H}^{+}$(NH\&3). Le

${ }^{4}$ L'antigène $A Q P I$ définit le groupe sanguin Colton. Son absence se traduit par un groupe $C O$ négatif. 
$\mathrm{Na}^{+}$est ensuite réabsorbé dans le milieu interstitiel grâce à la $\mathrm{Na}^{+} /$ $\mathrm{K}^{+}$-ATPase basolatérale.

À hauteur de l'anse de Henlé, la réabsorption de l'eau et du sodium est importante. Elle représente respectivement $15 \%$ et $30 \%$ de la charge filtrée. Pour comprendre son fonctionnement, il convient de diviser cette anse de Henlé en trois parties. Le premier segment est la partie descendante fine de l'anse : elle est globalement imperméable au $\mathrm{Na}^{+}$ mais très perméable à l'eau, via les $A Q P 1$. L'eau y est réabsorbée selon le gradient osmotique cortico-papillaire. Le second segment est la partie ascendante fine de l'anse qui est imperméable à l'eau mais permet une résorption passive de $\mathrm{Na}^{+}$. Le troisième et dernier segment est la branche ascendante large de l'anse qui est également imperméable à l'eau et assure une réabsorption active de $\mathrm{Na}^{+}$via le cotransporteur apical $\mathrm{Na}^{+} / \mathrm{K}^{+} / 2 \mathrm{Cl}^{-}$(NKCC2), avec, ensuite, extrusion basolatérale via la $\mathrm{Na}^{+} / \mathrm{K}^{+}$-ATPase. À noter que les diurétiques de l'anse, comme le furosémide ou le bumétanide, inhibent ce cotransporteur NKCC2.

Au niveau du tubule distal, le $\mathrm{Na}^{+}$est résorbé par le cotransporteur NCC ( $\mathrm{Na}^{+} / \mathrm{Cl}^{-}$co-transporter), sensible aux diurétiques thiazidiques. Du côté basolatéral, le $\mathrm{Na}^{+}$sort via la pompe $\mathrm{Na}^{+} / \mathrm{K}^{+}-$

$(\rightarrow)$ Voir la synthèse de C. Rafael et al., $m / s n^{\circ} 3$, mars 2016, page 274

ATPase [31] $(\rightarrow)$

Le canal collecteur assure moins de $5 \%$ de l'homéostasie hydro-sodée rénale. Cette fonction est cependant essentielle car elle est sujette à une régulation hormonale fine. Le canal collecteur est constitué de deux types cellulaires: les cellules intercalaires et les cellules principales. Les cellules intercalaires participent principalement à l'équilibre acidobasique de l'organisme. Les cellules principales interviennent quant à elles, dans l'homéostasie hydro-sodée. Ces cellules principales expriment le récepteur V2 de la vasopressine, également appelée «hormone antidiurétique » qui est produite par la neurohypophyse en réponse à un accroissement de l'osmolarité plasmatique ou une réduction du volume circulant. La liaison de la vasopressine au récepteur V2, couplé à la protéine Gs, induit la stimulation de l'AC, ce qui entraîne successivement l'augmentation de la synthèse d'AMPc, l'activation de la protéine kinase $A(P K A)$ et la phosphorylation des AQP2. Cette phosphorylation provoque leur trafic vers la membrane apicale et leur insertion. La PKA phosphoryle, en outre, la protéine CREB ( $C A M P$-response element-binding protein), ce qui augmente la transcription du gène $A Q P 2$. L'eau est ainsi réabsorbée grâce au gradient osmotique en passant dans les AQP2 apicales et les AQP3 et AQP4 basolatérales constitutives (Figure 1). La résorption sodée au niveau des cellules principales est, elle, contrôlée par l'aldostérone qui augmente la réabsorption du $\mathrm{Na}^{+}$via l'ENaC (epithelial sodium channel) et l'excrétion du potassium $\left(\mathrm{K}^{+}\right)$via ROMK (renal outer medullary potassium channel). Différents mécanismes autocrines et paracrines contrôlent les effets de la vasopressine et de l'aldostérone [16].

L'adénosine module la physiologie rénale comme elle le fait au niveau d'autres organes. Son rôle au niveau du rein est cependant complexe [17]. Dans le TCP, l'adénosine stimule la réabsorption du $\mathrm{Na}^{+}$via les récepteurs Al (Figure 1). Au niveau de la zone corticale du rein, elle intervient dans le rétrocontrôle tubulo-glomérulaire (RTG) via les récepteurs $A l$ en entraînant une vasoconstriction de l'artériole afférente en réponse à une augmentation de la concentration en $\mathrm{Na}^{+}$au niveau du TCD. Cette vasoconstriction entraîne une diminution du taux de filtration glomérulaire. L'implication des récepteurs $\mathrm{Al}$ dans cette réponse a été démontrée grâce à des expériences réalisées chez des souris invalidées pour le gène codant le récepteur $\mathrm{Al}[18,19]$. En revanche, l'adénosine augmente le flux sanguin au niveau de la zone médullaire via les récepteurs A2. Au niveau du canal collecteur, elle module l'effet de la vasopressine en exerçant un contrôle négatif sur l'AC et sur la production d'AMPc intracellulaire en se fixant à son récepteur $A l$, couplé aux protéines $\mathrm{Gi}$. Elle diminue dès lors l'activation de la PKA et la résorption de l'eau par ADP2 [17].

\section{Effet de la caféine au niveau des segments du néphron}

Étant donnée son action au niveau cardio-vasculaire, différents travaux ont porté sur l'impact de la caféine sur le débit sanguin rénal. Chez l'homme, aucune modification significative n'a pu être démontrée [11]. Néanmoins, il apparaît que le débit de filtration glomérulaire est accru par la consommation de caféine, malgré un débit plasmatique rénal conservé. Plusieurs études, réalisées chez l'animal, ont été entreprises afin de comprendre les mécanismes impliqués dans cet effet de la caféine et d'autres méthylxanthines. Ces études ont mis en évidence le rôle crucial du récepteur Al. En effet, l'administration de caféine n'a pas d'effet diurétique chez les souris déficientes en récepteur $A 1 R$ alors qu'elle provoque bien une augmentation du débit urinaire et de la natriurèse (voir ci-après) chez la souris sauvage [20]. Un des mécanismes proposés pour expliquer l'effet diurétique de la caféine est le blocage du rétrocontrôle tubulo-glomérulaire exercé par l'adénosine via ce récepteur Al. Par son effet antagoniste $\mathrm{Al}$, la caféine empêcherait en effet la vasoconstriction de l'artériole afférente en réponse à un débit accru d'ions $\left(\mathrm{Na}^{+}, \mathrm{Cl}^{-}\right.$et $\left.\mathrm{K}^{+}\right)$au niveau de la macula densa et entraînerait ainsi une augmentation du débit de filtration glomérulaire (Figure 1).

L'analyse d'urine récoltée après consommation de caféine, montre qu'elle est non seulement composée d'eau, mais également de $\mathrm{Na}^{+}$, de $\mathrm{Cl}^{-}$, de $\mathrm{Ca}^{2+}$, de $\mathrm{Mg}^{2+}$, de phosphate et d'autres solutés [21]. En particulier, la natriurèse augmenterait d'un facteur 1,5 après ingestion de $400 \mathrm{mg}$ de caféine [22]. L'inhibition de la réabsorption sodée par la caféine pourrait être expliquée par l'antagonisme du récepteur Al au niveau du TCP. Des expériences chez le rat montrent en effet que la caféine provoque rapidement une diminution d'expression de l'échangeur NHE3 (sodium-hydrogen exchanger 3) et de la $\mathrm{Na}^{+} / \mathrm{K}^{+}$-ATPase [23]. Cependant, des données 


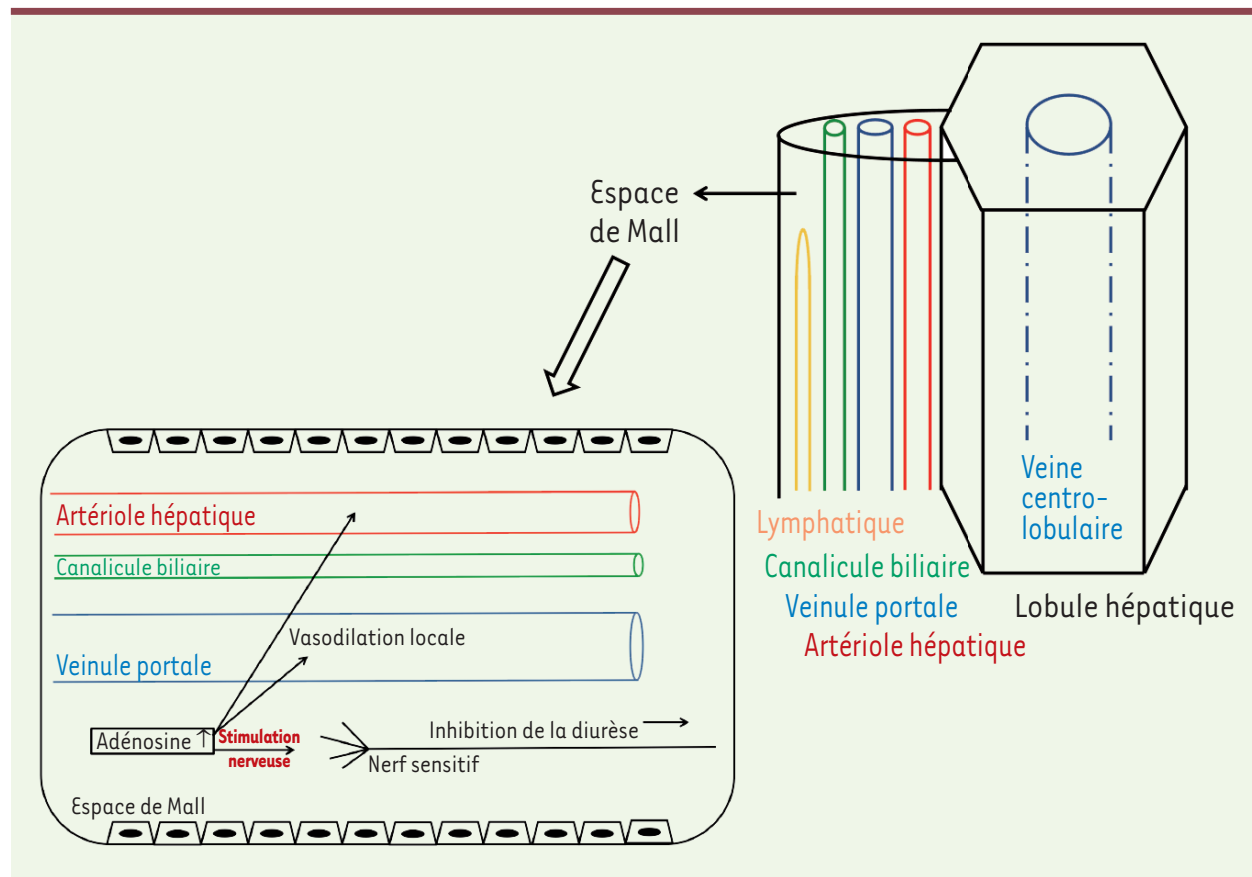

Figure 2. Représentation schématique de l'espace intra-hépatique de Mall et du réflexe hépato-rénal contrôlé par l'adénosine. L'adénosine est constamment sécrétée dans les espaces hépatiques de Mall, espaces péri-lobulaires entourant les branches terminales des artérioles hépatiques, les veinules portales et les différents constituants de l'espace porte. La concentration locale d'adénosine est maintenue constante grâce au flux sanguin. Elle augmente dès que le flux portal diminue. Une concentration majorée d'adénosine non seulement induit une vasodilatation locale de la veinule portale et de l'artériole hépatique, mais serait également détectée par les terminaisons nerveuses qui induiraient un réflexe hépatorénal visant à diminuer les pertes urinaires d'eau et de sel.

récentes montrent que les souris déficientes en NHE3 au niveau du TCP répondent à l'administration de caféine par une augmentation de la diurèse et de la natriurèse qui est similaire aux souris contrôles, ce qui suggère l'implication d'autres transporteurs. En particulier, l'inhibition des co-transporteurs $\mathrm{Na}^{+} / \mathrm{HCO}^{-}$et $\mathrm{Na}^{+} /$phosphate participerait à cet effet natrio-diurétique induit par la caféine [24].

Outre cet effet natriurétique, il existe d'autres arguments en faveur d'une diurèse tubulaire proximale induite par la caféine. Sous caféine, on observe notamment un accroissement de la calciurie de débit (mesurée en $\mathrm{mmol} / \mathrm{kg} / \mathrm{jour}$ ) [20]. Cette perte calcique a des conséquences sur l'homéostasie minérale, avec une élévation des taux de parathormone (PTH) chez les grands consommateurs de café dont les apports calciques sont faibles [25, 26]. La clairance du lithium, qui reflète la diminution de réabsorption tubulaire proximale de $\mathrm{Na}^{+}$, est également accrue par la caféine [22]. Dès lors, une augmentation des taux plasmatiques de lithium peut s'observer lors de l'arrêt d'une consommation chronique de café [27].

Au niveau du canal collecteur, la caféine n'aurait pas d'effet significatif sur les récepteurs $\mathrm{Al}$ des cellules principales. L'inhibition des phosphodiestérases y serait par ailleurs nulle aux concentrations circulantes usuelles de méthylxanthines chez l'homme. L'implication des récepteurs A2a demeure actuellement incertaine.

\section{Effet de la caféine sur le réflexe hépatorénal}

Outre l'impact de la caféine sur le réflexe tubulo-glomérulaire (RTG) et la fonction du TCP conduisant à une natriurèse et une diurèse accrues, un tout autre mécanisme physiologique a été décrit in vivo chez le rat. II implique le réflexe hépatorénal. Au niveau hépatique, l'adénosine est constamment sécrétée dans les espaces de Mall ${ }^{5}$. À ce niveau, sa concentration est déterminée par le flux sanguin : elle augmente dès que le flux portal diminue. Une concentration accrue d'adénosine dans les espaces de Mall induirait non seulement une vasodilatation de l'artère hépatique via les récepteurs $A l$, mais serait également responsable d'un réflexe hépatorénal. En effet, la stimulation des terminaisons nerveuses des espaces de Mall par l'adénosine provoquerait une diminution des pertes urinaires d'eau et de sel afin de restaurer un meilleur flux sanguin au niveau hépatique [28] (Figure 2). Chez l'animal, l'injection intra-portale d'adénosine suivie d'une injection de caféine provoque l'arrêt immédiat de l'effet anti-natriurétique et anti-diurétique de l'adénosine. De plus, l'injection de $5 \mathrm{mg} / \mathrm{kg}$ de caféine en intra-portal induit une augmentation du débit urinaire de $82 \%$ chez des rats sains. Ce réflexe est perdu en cas de dénervation hépatique [29].

Ces observations réalisées chez le rat suggèrent que la rétention hydro-sodée induite lors de pathologies hépatiques pourrait être corrigée par un antagonisme Al au niveau hépatique. En effet, un foie malade libérerait de l'adénosine dans les espaces de Mall par une dégradation d'adénine secondaire à la réaction inflammatoire. La caféine pourrait dès lors être un candidat pharmacologique pour le traitement du syndrome hépatorénal. Cependant, ses propriétés pharmacocinétiques

${ }^{5}$ Les espaces de Mall correspondent aux zones péri-lobulaires entourant les branches terminales des artérioles hépatiques, les veinules portales et les différents constituants de l'espace porte. Ils sont situés dans la prolongation des espaces péri-sinusoïdaux de Disse. Leur fonction est de contenir l'excès de liquide sécrété à ce niveau avant qu'il ne soit repris par les canaux lymphatiques. 
(en particulier sa courte demi-vie) n'en font pas une molécule idéale. Des essais cliniques utilisant des antagonistes synthétiques des récepteurs Al (mimant les effets de la caféine) sont actuellement en évaluation pour le traitement de la rétention d'eau en cas d'insuffisance hépatique, et dans le traitement de l'insuffisance cardiaque [29, 30$]$.

\section{Conclusion}

L'effet diurétique du café est bien connu des consommateurs. Bien qu'on ne puisse exclure le rôle de substances autres que les méthylxanthines, présentes dans le café, la pharmacologie de cet effet diurétique semble principalement médiée par la caféine et impliquerait les récepteurs de l'adénosine. Notre revue de la littérature suggère que le rein n'est pas le seul organe impliqué mais que le foie pourrait également participer à l'effet natriurétique et diurétique de la caféine. Au niveau du rein, deux mécanismes principaux interviennent : le blocage du rétrocontrôle tubulo-glomérulaire et l'inhibition de la réabsorption sodée par le TCP. Au niveau du foie, la caféine s'opposerait au réflexe hépatorénal induit par l'adénosine intra-hépatique. Ces effets synergiques résultent en une augmentation de la diurèse et de la natriurèse. $\diamond$

\section{SUMMARY}

\section{Mechanisms of caffeine-induced diuresis}

Caffeine is an alkaloid which belongs to the family of methylxanthines and is present in beverages, food and drugs. Caffeine competitively antagonizes the adenosine receptors (AR), which are $G$ protein-coupled receptors largely distributed throughout the body, including brain, heart, vessels and kidneys. Caffeine consumption has a well-known diuretic effect. The homeostasis of salt and water involves different segments of the nephron, in which adenosine plays complex roles depending on the differential expression of AR. Hence, caffeine increases glomerular filtration rate by opposing the vasoconstriction of renal afferent arteriole mediated by adenosine via type 1 AR during the tubuloglomerular feedback. Caffeine also inhibits $\mathrm{Na}^{+}$reabsorption at the level of renal proximal tubules. In addition, caffeine perturbs the hepatorenal reflex via sensory nerves in Mall's intrahepatic spaces. Here, we review the physiology of caffeine-induced natriuresis and diuresis, as well as the putative pathological implications. $\diamond$

\section{REMERCIEMENTS}

Les auteurs remercient vivement Laurent Massotte pour son aide graphique dans la réalisation des figures.

\section{LIENS D'INTÉRÊT}

Les auteurs déclarent n'avoir aucun lien d'intérêt concernant les données publiées dans cet article.

\section{RéFÉRENCES}

1. Fredholm BB, Bättig K, Holmén J, et al. Actions of caffeine in the brain with special reference to factors that contribute to its widespread use. Pharmacol Rev $1999 ; 51: 83-133$.

2. Chen JF, Eltzschig HK, Fredholm BB. Adenosine receptors as drug targets: what are the challenges? Nat Rev Drug Discov $2013 ; 12: 265-86$.

3. Sawynok J. Pharmacological rationale for the clinical use of caffeine. Drugs $1995 ; 49: 37-50$.

4. Fuxe K, Ferré $S$, Genedani $S$, et al. Adenosine receptor-dopamine receptor interactions in the basal ganglia and their relevance for brain function. Physiol Behav $2007 ; 92: 210-7$.
5. El Yacoubi M, Ledent C, Parmentier M, et al. Reduced appetite for caffeine in adenosine A2A receptor knockout mice. EurJ Pharmacol $2005 ; 519: 290-1$.

6. Riksen NP, Smits P, Rongen GA. The cardiovascular effects of methylxanthines. Handb Exp Pharmacol $2011 ; 200: 413-37$.

7. Tabrizchi R, Bedi S. Pharmacology of adenosine receptors in the vasculature. Pharmacol Ther $2001 ; 91: 133-47$.

8. Brown RA, Spina D, Page (P. Adenosine receptors and asthma. $\mathrm{Br}$ ) Pharmacol 2008 ; 153 : S446-56.

9. Boekema PJ, Samsom M, van Berge Henegouwen GP, Smout AJ. Coffee and gastrointestinal function: facts and fiction. A review. Scand J Gastroenterol Suppl $1999 ; 230: 35-39$.

10. Hodgson AB, Randell RK, Jeukendrup AE. The metabolic and performance effects of caffeine compared to coffee during endurance exercise. PLoS One $2013 ; 8$ : e59561.

11. Passmore AP, Kondowe GB, Johnston GD. Renal and cardiovascular effects of caffeine: a dose-response study. Clin Sci (Lond) 1987 ; 72 : 749-56.

12. Maughan RJ, Griffin J. Caffeine ingestion and fluid balance: a review. J Hum Nutr Diet $2003 ; 16: 411-20$.

13. Neuhäuser-Berthold, Beine S, Verwied SC, Lührmann PM. Coffee consumption and total body water homeostasis as measured by fluid balance and bioelectrical impedance analysis. Ann Nutr Metab 1997 ; 41 : 29-36.

14. Izzo JL Jr, Ghosal A, Kwong T, et al. Age and prior caffeine use alter the cardiovascular and adrenomedullary responses to oral caffeine. Am J Cardiol $1983 ; 52: 769-73$.

15. Greger R. Physiology of renal sodium transport. Am J Med Sci 2000 ; 319: 51-62.

16. Pearce D, Soundararajan R, Trimpert C, et al. Collecting duct principal cell transport processes and their regulation. Clin J Am Soc Nephrol $2015 ; 10$ : 135-46.

17. Vallon V, Osswald $\mathrm{H}$. Adenosine receptors and the kidney. Handb Exp Pharmacol $2009 ; 193: 443-70$.

18. Brown $R$, Ollerstam $A$, Johansson $B$, et al. Abolished tubuloglomerular feedback and increased plasma renin in adenosine Al receptor-deficient mice. Am J Physiol Regul Integr Comp Physiol 2001 ; 281 : R1362-7.

19. Sun D, Samuelson LC, Yang T, et al. Mediation of tubuloglomerular feedback by adenosine: evidence from mice lacking adenosine 1 receptors. Proc Natl Acad Sci USA 2001 ; 98 : 9983-8.

20. Rieg T, Steigele H, Schnermann J, et al. Requirement of intact adenosine $\mathrm{Al}$ receptors for the diuretic and natriuretic action of the methylxanthines theophylline and caffeine.J Pharmacol Exp Ther $2005 ; 313: 403-9$.

21. Osswald H, Schnermann J. Methylxanthines and the kidney. Handb Exp Pharmacol $2011 ; 200: 391-412$.

22. Shirley DG, Walter SJ, Noormohamed FH. Natriuretic effect of caffeine: assessment of segmental sodium reabsorption in humans. Clin Sci (Lond) $2002 ; 103: 461-6$.

23. Lee J, $\mathrm{Ha} \mathrm{JH,} \mathrm{Kim} \mathrm{S,} \mathrm{et} \mathrm{al.} \mathrm{Caffeine} \mathrm{decreases} \mathrm{the} \mathrm{expression} \mathrm{of} \mathrm{Na}^{+} /$ $\mathrm{K}^{+}$-ATPase and the type $3 \mathrm{Na}^{+} / \mathrm{H}^{+}$exchanger in rat kidney. Clin $\operatorname{Exp}$ Pharmacol Physiol $2002 ; 29$ : 559-63.

24. Fenton RA, Poulsen SB, de la Mora Chavez S, et al. Caffeine-induced diuresis and natriuresis is independent of renal tubular NHE3. Am J Physiol Renal Physiol 2015 ; 308 : F1409-20.

25. Massey LK, Bergman EA, Wise KJ, Sherrard DJ. Interactions between dietary caffeine and calcium on calcium and bone metabolism in older women. J Am Coll Nutr $1994 ; 13: 592-6$.

26. Wise KJ, Bergman EA, Sherrard DJ, Massey LK. Interactions between dietary calcium and caffeine consumption on calcium metabolism in hypertensive humans. Am J Hypertens $1996 ; 9: 223-9$.

27. Mester R, Toren P, Mizrachi I, et al. Caffeine withdrawal increases lithium blood levels. Biol Psychiatry $1995 ; 37$ : 348-50.

28. Ming Z, Smyth DD, Lautt WW. Decreases in portal flow trigger a hepatorenal reflex to inhibit renal sodium and water excretion in rats: role of adenosine. Hepatology $2002 ; 35: 167-75$.

29. Ming Z, Lautt WW. Caffeine-induced natriuresis and diuresis via blockade of hepatic adenosine-mediated sensory nerves and a hepatorenal reflex. Can J Physiol Pharmacol $2010 ; 88$ : 1115-21.

30. Møller S, Bendtsen F, Henriksen JH. Pathophysiological basis of pharmacotherapy in the hepatorenal syndrome. Scand J Gastroenterol 2005 ; $40: 491-500$.

31. Rafael C, Chavez-Canales M, Hadchouel J. Une vision nouvelle du rôle de WNKl et WNK4 dans la régulation de la réabsorption de $\mathrm{NaCl}$ et la sécrétion de potassium par le néphron distal. Med Sci (Paris) 2016 ; 32 : 274-80.

\section{TIRÉS À PART}

F. Jouret 